\title{
CURVATURE AND TEMPERATURE EFFECT ON $n$-DECANE TRANSPORT IN NARROW CARBON NANOTUBES
}

\author{
ZHONGLIANG CHEN ${ }^{1 *}$, XIAOHU DONG ${ }^{1} \&$ ZHANGXIN CHEN $^{2}$ \\ ${ }^{1}$ State Key Laboratory of Petroleum Resources and Prospecting, China University of Petroleum, China \\ ${ }^{2}$ Department of Chemical and Petroleum Engineering, University of Calgary, Canada
}

\begin{abstract}
Carbon nanotubes (CNTs) are excellent materials for advanced functional nano-elements. They have superior mechanical, electronic, and chemical properties and are widely used in many fields, such as nanomechanics, advanced electronics, biotechnology, and energy. Alkanes transport through CNTs has received widespread attention. This study aims to propose a systematic method to study the coupling effect of curvature and temperature on the $n$-decane transport through narrow CNTs. The OPLS (optimized potentials for liquid simulations) model and Lennard-Jones potential are used to describe the intermolecular/intramolecular interactions in a typical $n$-decane/CNT system. All molecular dynamics (MD) simulations are conducted in the NVT ensemble to show the dynamic of $n$-decane molecules in 1.08, 1.36, and $2.71 \mathrm{~nm}$-diameter single-walled armchair CNTs. The Green-Kubo and Stokes-Einstein expression are combined with the MD simulations to calculate the $n$-decane/CNT friction coefficient, the $n$-decane axial self-diffusion coefficient, and viscosity in CNTs. The results show that increased curvature causes the $n$-decane/CNT friction coefficient to decline rapidly. However, the changes in the axial self-diffusion coefficient and viscosity are non-monotonic. On the contrary, the effect of increasing temperature is just the opposite; that is, for individual CNTs, the axial self-diffusion coefficient generally increases, and the viscosity decreases, but the friction coefficient fluctuates. We also find that the non-monotonic change between the curvature and the axial selfdiffusion coefficient is substantially temperature-independent. An increase in temperature has a positive effect on the axial diffusion of $n$-decane molecules. However, when the curvature of carbon nanotubes is too large (the $1.08 \mathrm{~nm}$-diameter CNT), there is no way to sustain this positive effect. It is worth emphasizing that even with high temperatures, a CNT with a more significant curvature does not mean that $n$-decane is more difficult to transport through.
\end{abstract}

Keywords: carbon nanotube, curvature, $n$-decane, friction coefficient, diffusion.

\section{INTRODUCTION}

Nanofluidics is a study for the behaviour, manipulation and control of fluids that are confined to nanostructures. This process has experienced considerable growth in recent years [1]. Due to the characteristic physical scaling lengths of fluid closely coincide with the dimensions of the nanostructure itself, new solutions and properties can be obtained from the scales where the behavior of matter departs from conventional expectations [2], [3]. The spatial structure of the forces acting on the nanoscale must be fully taken into account to understand how fluids behave [4].

CNTs are excellent materials for advanced functional nano-elements. They have superior mechanical, electronic and chemical properties, and are widely used in many fields [5], [6], such as nanomechanics, advanced electronics, biotechnology and energy. MD simulations, acting as a bridge between microscopic length and time scales and the macroscopic world of laboratory [7], allow us to focus on the dynamical properties of a typical fluid/CNT system,

* ORCID: https://orcid.org/0000-0002-8881-2294 
such as transport coefficients, time-dependent responses to perturbations and rheological properties.

As an intrinsically interfacial property, the inverse hyperbolic sine relationship [8] shows that the friction dominates the transport behaviour of water at the wall of CNT. Even increasing the interactions between water and CNT by modifying the membranes, it still produces non-uniform nanofluid flow with lower friction than that consistent with the Navier-Stokes equations [9]. Once we calculate the activation energy required for the transportation process, we can interpret the transport behaviour and predict the friction coefficient [10] between water and CNT. However, it is slightly different from the results [11] determined using the Green-Kubo relationship of the liquid-solid friction coefficient. There is also a significant flow enhancement for decane through nanoscale CNTs [12], which can be explained by the lower friction coefficient at carbon interface. The flow rate of decane is four to five orders of magnitude faster than conventional fluid flow would predict through $7 \mathrm{~nm}$-diameter CNT [13], and the observed slip length $(3.4 \mu \mathrm{m})$ are much longer than the pore radius $(3.5 \mathrm{~nm})$ that is consistent with a nearly frictionless interface.

The values of water self-diffusion coefficient vary widely in different CNTs, depending on the density, temperature, and confinement [14]. However, for a set of narrow CNTs with the same cross-sectional area but different cross-sectional shapes, the mobilities of water can also differ considerably [15]. Furthermore, it is possible to separate the effect of the CNT surface and the effect of the cross-sectional shape of the confinement [16], and the diffusion mechanisms of ballistic, Fickian and single-file types can be determined by analyzing the style of the time evolution of the mean squared displacement [17]. For $n$-decane molecules, the anomalous positive peaks are observed in the velocity autocorrelation function perpendicular to the axis of CNT, which can be explained by the oscillating motion of the molecules trapped in the effective potential well produced by the wall of CNT [18], and the diffusion of molecules in this area is different from that in the central area of CNT.

Viscosity is a physical property of a fluid that opposes the relative motion between two liquid layers, or in a fluid that moves at different velocities [19]. For a larger set of CNTs, the viscosity of the confined water increases with the increase of temperature and CNT diameter, while the size-dependent trend of viscosity is almost independent of temperature [20]. When we consider a single file or a single layer of molecules transport through a narrow CNT, the calculation of viscosity based on the Eyring theory of reaction rates seems not suitable for this case [21]. However, the equilibrium molecular dynamics simulation does not require other adjustments that the nonequilibrium method usually needs, and has shown advantages in solving such problems [22]. Although Einstein's model [23] does not include the structural parameters of the flow channel [24], the diffusion coefficient in this model is very sensitive to the curvature [25] and even flexibility [26] of the CNTs.

The main goal of this paper is to propose a systematic method to study the coupling effect of curvature and temperature on the $n$-decane transport through narrow CNTs. The focus is two-fold. First, the combination of theory and MD simulations is emphasized, including the theoretical background of the friction coefficient and transport coefficient based on GreenKubo expression and the Stokes-Einstein relationship, as well as the process of model building and the selection of essential parameters. Second, focus on the core of the subject, which is to use MD simulation to understand how curvature and temperature affect the transport of $n$-decane in narrow CNTs. Although some notions are at the historical foundation of the subject, new systematic practice and exciting results of MD simulations have recently come to light. 


\section{THEORY AND MD SIMULATION}

\subsection{Liquid-solid friction coefficient}

Liquid-solid friction coefficient affects the fluid flow and permeability of porous media, which is often used as an essential parameter in models of transport properties through microand nano-channels. A geometry model of the Brownian motion of a cylindrical wall (CNT) in contact with a liquid ( $n$-decane) is introduced and a non-Marconian Langevin equation (eqn (1)) of motion of the fluctuating wall [27], including the liquid-solid friction coefficient, $\lambda$, is established.

$$
m \frac{d U}{d t}=-\lambda A v_{s}(t)+\delta F(t)
$$

In eqn (1), $A$ is the lateral surface area, $\delta F(\mathrm{t})$ is the lateral fluctuating force, $m$ and $U(\mathrm{t})$ are the mass and velocity of the wall, respectively. The hydrodynamic slip velocity, $v_{\mathrm{s}}(\mathrm{t})$, which describes the velocity discontinuity between the wall and the liquid, can be defined as [27],

$$
v_{s}(t)=\int_{-\infty}^{+\infty} d t^{\prime} K\left(t-t^{\prime}\right) U\left(t^{\prime}\right)
$$

where, $K(\mathrm{t})$ is the memory kernel. The introduction of the memory kernel with vanishing time integral allows us to have a better acquaintance with the real slip velocity in the linear response regime. We use the Laplace transform of the velocity autocorrelation function $C(\mathrm{t})$ $=<U(\mathrm{t}) U(0)>$ and the sum rules of the memory kernel to calculate the Laplace transform of the autocorrelation function of the axial component of the force acting on the lateral surface [27]. The Green-Kubo relation for the friction coefficient is found by integrating the force autocorrelation function over time [27],

$$
\lambda=\frac{1}{A k_{B} T} \int_{0}^{\infty} d t\left\langle F_{z}(t) \cdot F_{z}(0)\right\rangle,
$$

where, $k_{B}$ is the Boltzmann constant and $T$ is the temperature.

\subsection{Diffusion coefficient and viscosity}

The axial self-diffusion coefficient [28], [29] (in one dimension), $D_{z}$, valid at long times, can be written as,

$$
D_{z}=\lim _{t \rightarrow \infty} \frac{d}{d t} \frac{1}{2}\left\langle|z(t)-z(0)|^{2}\right\rangle=\lim _{t \rightarrow \infty} \frac{d}{d t} \frac{1}{2}\left\langle\frac{1}{N} \sum_{i=1}^{N}\left|z_{i}(t)-z_{i}(0)\right|^{2}\right\rangle .
$$

For each of the $N$ atoms in the simulation, the centre-of-mass axial position is $z_{\mathrm{i}},<\mid z(\mathrm{t})$ $\left.z(0)\right|^{2}>$ is the mean squared displacement (MSD) [30], [31].

And the corresponding shear viscosity, $\eta$, is found to be proportional to the mean squared $x$ displacement of the centre of $y$ momentum [30], [32], [33].

$$
\eta=\lim _{t \rightarrow \infty} \frac{d}{d t} \frac{1}{2} \frac{V}{k_{B} T}\left\langle\left[Q_{x y}(t)-Q_{x y}(0)\right]^{2}\right\rangle,
$$


where $V$ is a volume of the particle system and the dynamical variable, $Q_{\mathrm{xy}}$, can be defined as,

$$
Q_{x y}=\frac{1}{V} \sum_{i=1}^{N} x_{i} m_{i} v_{i y}
$$

where $m_{i}, x_{i}$ and $v_{i y}$ are the mass, the centre-of-mass radial position and velocity for each of the $N$ atoms, respectively.

Obviously, given the existing axial self-diffusion coefficient, $D_{z}$, we also can predict the viscosity by evoking the Stokes-Einstein, SE, relationship [28], i.e. the theory of Brownian motion for a particle slightly more massive than or approximately equal to the solvent molecules [34], [35], of diameter $2 r$, immersed in a liquid leads to a relationship [36] between the shear viscosity, $\eta$, of the host liquid and the axial self-diffusion coefficient, $D_{z}$, of the particle,

$$
D_{z}=\frac{k_{B} T}{4 \pi \eta r}
$$

where, the effective diameter [34] for an $n$-decane molecule is $4.2 \AA$.

\subsection{Simulation parameters and model}

$n$-Decane interactions are modelled using the OPLS (optimized potentials for liquid simulations) model [37], $\mathrm{CH}_{n}$ groups are treated as united atoms centered on the carbon [38], [39], i.e. every methyl $\left(\mathrm{CH}_{3}\right)$ or methylene $\left(\mathrm{CH}_{2}\right)$ group is modelled as a single interaction site [40]. The equilibrium value of the distance between neighboring sites [39], [41] (the bond length) is $1.53 \AA$, the equilibrium value of the angle between two connected bonds [39], [42] (the bond angle) is $112^{\circ}$, and the harmonic force constants for bonds [41], [43] and angles [11], [44] are $900 \mathrm{kcal} / \mathrm{mol} / \AA$ and $124.2 \mathrm{kcal} / \mathrm{mol} / \mathrm{rad}^{2}$, respectively. The Fourier series (eqn (8)) can describe the rotational potential energy [37] and let the dihedral angle C-C-C-C vary over a sufficiently broad range [39].

$$
V(\phi)=\frac{1}{2} \times 1.411 \times(1+\cos \phi)-\frac{1}{2} \times 0.271 \times(1-\cos 2 \phi)+\frac{1}{2} \times 3.145 \times(1+\cos 3 \phi) .
$$

The Lennard-Jones potential suffices to describe the non-bonded interactions between the united atoms from different molecules and within a molecule [40] (if two atoms are more than four atoms apart), and the corresponding optimized Lennard-Jones parameters are listed in Table 1. A standard mixing rule, $\varepsilon_{i j}=\sqrt{\varepsilon_{i} \varepsilon_{j}}$ and $\sigma_{i j}=\frac{1}{2}\left(\sigma_{i}+\sigma_{j}\right)$, is used to create pair coefficients for interactions between different (united) atoms.

Table 1: Optimized Lennard-Jones parameters for $n$-decane (XX) and $n$-decane-CNT (XC) interaction.

\begin{tabular}{|c|c|c|c|c|}
\hline $\mathrm{X}$ & $\sigma_{\mathrm{XX}}(\AA)$ & $\varepsilon_{\mathrm{XX}}(\mathrm{kcal} / \mathrm{mol})$ & $\sigma_{\mathrm{XC}}(\AA)$ & $\varepsilon_{\mathrm{XC}}(\mathrm{kcal} / \mathrm{mol})$ \\
\hline $\mathrm{CH}_{3}$ & 3.905 & 0.175 & 3.828 & 0.136 \\
\hline $\mathrm{CH}_{2}$ & 3.905 & 0.118 & 3.828 & 0.111 \\
\hline
\end{tabular}


The nanotube-builder for VMD [45] is used to generate 1.08, 1.36, and $2.71 \mathrm{~nm}$-diameter single-walled armchair CNTs with smooth tips, all of which are 10 nanometers long. We do not plan on defining bonded interactions between carbon atoms. A snapshot [45] from a typical $n$-decane/CNT system is presented in Fig. 1 , and the chirality vector for each CNT is listed.

(a)

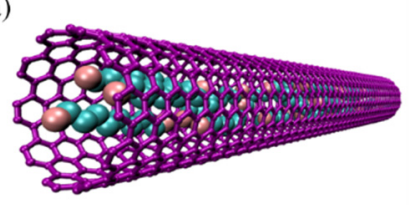

(b)

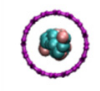

$(08,08)$ (c)

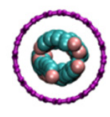

$(10,10)$ (d)

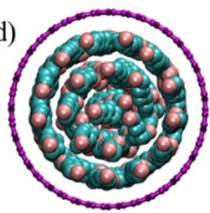

$(20,20)$

Figure 1: (a) A snapshot from a typical $n$-decane/CNT system. The diameters of the singlewalled armchair CNTs are $1.08 \mathrm{~nm}(\mathrm{~b}), 1.36 \mathrm{~nm}$ (c) and $2.71 \mathrm{~nm}$ (d) respectively.

$n$-Decane-carbon friction and $n$-decane diffusion coefficient can be predicted by using a Green-Kubo relation in an equilibrium (no net flow) simulation [11], [27], but until then, an additional equilibrium simulation [11] is needed to push $n$-decane molecules inside the tube using a piston-like mechanism. The pressure applied to the piston is one atmosphere [39], which ensures that there are a suitable number of $n$-decane molecules in CNTs of different sizes. After reaching equilibrium, the pistons and $n$-decane reservoirs on both sides of the CNT are removed.

All simulations are performed in the NVT ensemble (constant mass, volume, and temperature) with a temperature maintained at a specific value using a Nosé-Hoover thermostat [46], time integration is performed on Nosé-Hoover style non-Hamiltonian equations of motion which are designed to generate positions and velocities sampled from the canonical ensemble. The temperature is relaxed in a time span of 0.2 ps [39] in anticipation of a balance between milder temperature fluctuations and less equilibration time. In order to guarantee the quality of the equilibrium simulations, the temperature is changed from $300 \mathrm{~K}$ to $360 \mathrm{~K}$ by $3 \mathrm{~K}$ steps, using the final configuration from the previous temperature as initial state [47].

The total force between the $\mathrm{CH}_{n}$ groups and the carbon atoms is measured every 2 fs [39] to calculate the autocorrelation function of the axial component of the force acting on the wall of the CNT (eqn (3)). Once the linear state in MSD (eqn (4)) is reached, the axial selfdiffusion coefficient can be better estimated at an early stage rather than at later correlation times [48]. Successive time origins [49] are set to produce the Green-Kubo curves and the MSD curves, adequate statistics and the standard error of the estimate are used [47], [49] for determination of the $n$-decane/CNT friction coefficient and the $n$-decane axial self-diffusion coefficient, error bars in the simulations are roughly similar or smaller than the symbols in the following figures. 


\section{RESULTS AND DISCUSSION}

\section{$3.1 n$-Decane-carbon friction coefficient}

As shown in Fig. 2, our MD simulation results for the $n$-decane/CNT friction coefficient are consistent with the equilibrium and flow MD simulation results in the published paper [39]. The results of the water/CNT friction coefficient [39] are also shown for comparison. Interestingly, although the structure of the $n$-decane molecule is larger and more complicated than that of water molecules, the $n$-decane/CNT friction coefficient is smaller. And they all decrease with the increase in curvature.

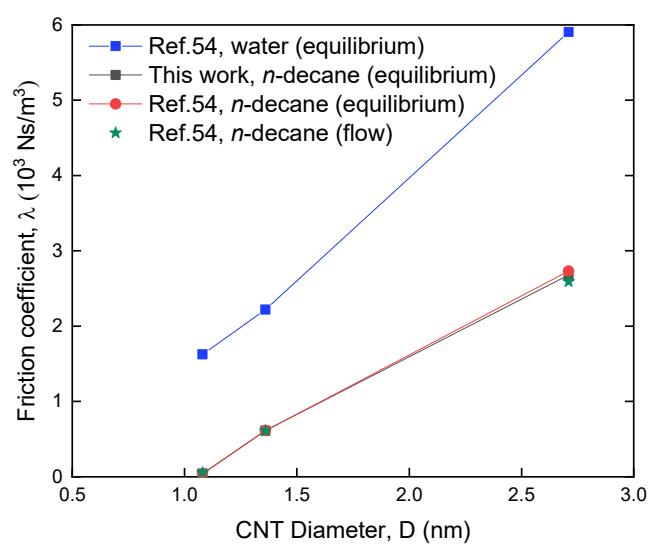

Figure 2: Variati on in water/CNT and $n$-decane/CNT friction coefficient with CNT diameter $D$. We compared the MD results of the $n$-decane/CNT friction coefficient (eqn (3)) with the published results [39], including the equilibrium and nonequilibrium (flow) MD simulations. The water/CNT friction coefficient [39] is also shown as a reference. All of these simulations have a temperature of $300 \mathrm{~K}$.

We also find that this trend remains the same, even when affected by temperature-related changes (Fig. 3(a)). But if we look more closely, the plot actually thickens (Fig. 3(b)), i.e. for individual CNTs, the change of the curve is non-monotonous due to the coupling effect of curvature and temperature.

The friction coefficient fluctuates more dramatically in smaller CNTs. For the $2.71 \mathrm{~nm}$ diameter single-walled armchair CNT, raising the temperature generally reduce the friction coefficient, which is quite the opposite for the smallest CNT, and the situation is somewhere in between in the $1.36 \mathrm{~nm}$-diameter CNT.

\section{$3.2 n$-Decane diffusion coefficient}

We can think of $n$-decane as an ellipsoid [50], and if the rotation of the uniaxial anisotropic particle is prohibited, its diffusion will mainly occur in the direction parallel to its long axis [51], but even if the rotation is permitted, according to the results [52] obtained using the diffusion map approach to observe such molecules with high aspect ratios in aqueous 


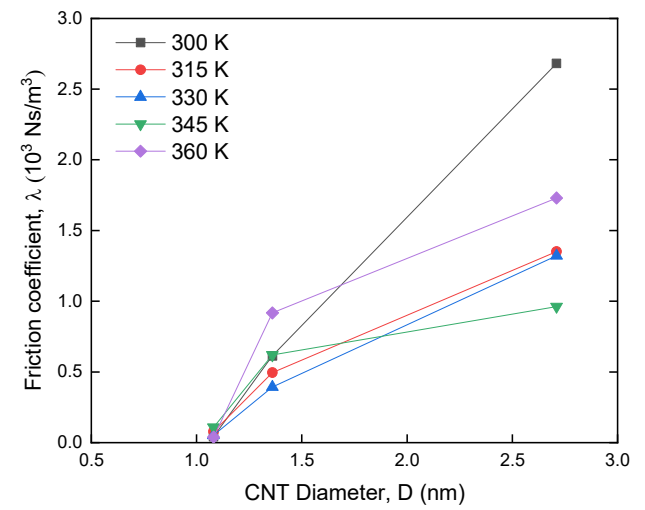

(a)

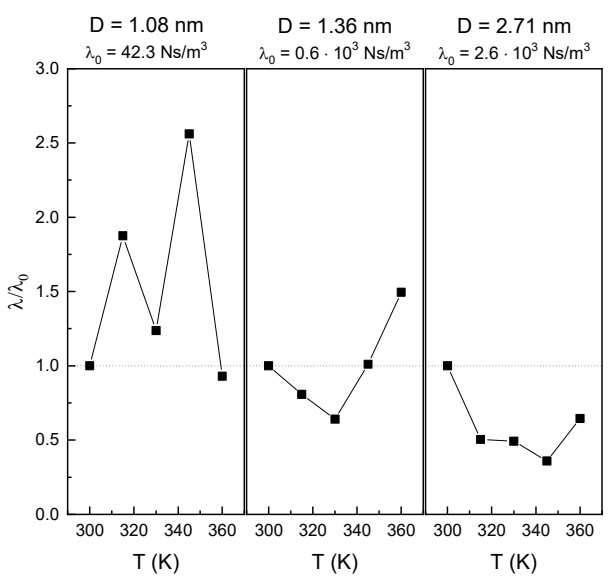

(b)

Figure 3: Curvature and temperature effect on $n$-decane/CNT friction coefficient, as predicted from equilibrium MD simulation using eqn (3). (a) Friction coefficient versus CNT diameter; and (b) Friction coefficient versus temperature, $\lambda_{0}$ is the $n$-decane/CNT friction coefficient at $300 \mathrm{~K}$.

solution, as one of the ordered principal moments, $\zeta_{1}$ describes the extent of the molecule along its longest axis and contributes the most to the radius of gyration.

If we go the extra mile and go from aqueous solution to CNTs (Fig. 4), we will find that $n$-decane molecules tend to line along the axial direction in CNTs, such a structural arrangement (i.e. the orientational ordering) would lead to a relatively small displacement in the radial direction [11], [53].

(a)

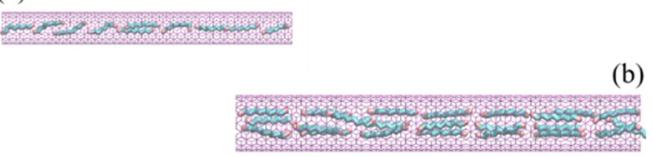

(c)

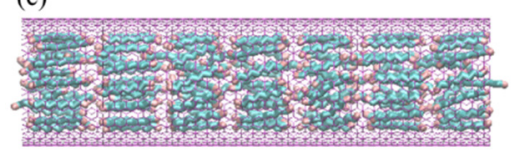

Figure 4: The structural arrangement of $n$-decane molecules in CNTs. The diameters of the single-walled armchair CNTs are $1.08 \mathrm{~nm}$ (a), $1.36 \mathrm{~nm}$ (b) and $2.71 \mathrm{~nm}$ (c) respectively.

Different from the calculation results of the friction coefficient, the axial self-diffusion coefficient of $n$-decane does not show a monotonic change with the decreasing diameter of CNTs (Fig. 5(a)). We believe that this non-monotonicity is the result of the tripartite game among the curvature, the depletion area [11], [54] at the $n$-decane/CNT interface and the central area. 


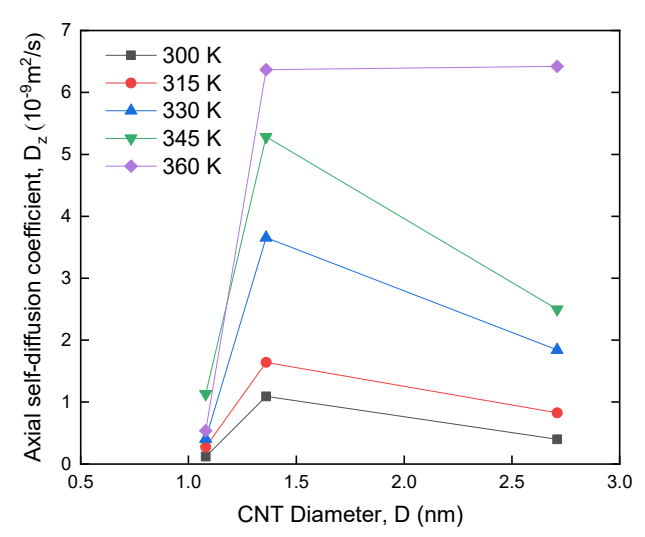

(a)

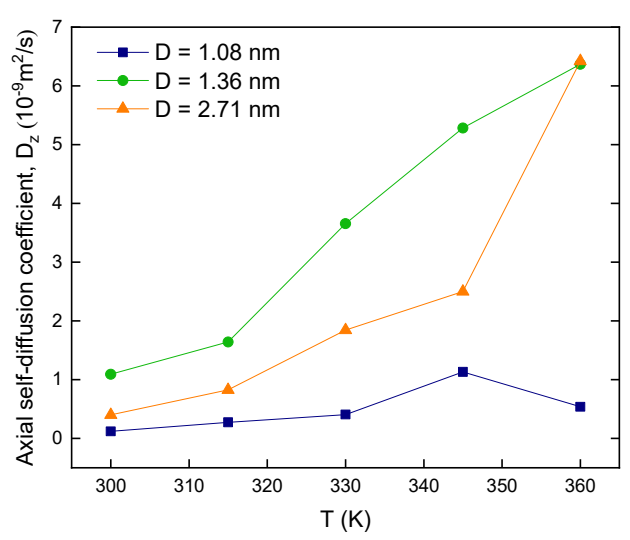

(b)

Figure 5: Curvature and temperature effect on axial self-diffusion coefficient of $n$-decane, as predicted from equilibrium MD simulation using eqn (4). (a) Axial selfdiffusion coefficient versus CNT diameter; and (b) Axial self-diffusion coefficient versus temperature.

For the $1.36 \mathrm{~nm}$-diameter single-walled armchair CNT, a depletion area at the $n$ decane/CNT interface plays a dominant role in affecting the axial diffusion of $n$-decane molecules, which can be shown by the complexity of the spatial variations (from the centre to the nanotube wall) [55] in the axial self-diffusion coefficient, i.e. the more significant the axial diffusion in the area closer to the wall of CNTs.

For the $1.08 \mathrm{~nm}$-diameter CNT, excessive curvature is primarily responsible for the reduction of the axial self-diffusion coefficient of $n$-decane molecules. However, for the $2.71 \mathrm{~nm}$-diameter CNT, the effects from curvature and depletion area are more or less weakened, and the bulk diffusion of the central area becomes evident.

Further shifting the focus to changes in temperature, we find that this non-monotonic change between the curvature and the axial self-diffusion coefficient is substantially temperature-independent (Fig. 5(a)). For individual CNTs, an increase in temperature generally has a positive effect on the axial diffusion of molecules (Fig. 5(b)). However, when the curvature of carbon nanotubes is too large (the $1.08 \mathrm{~nm}$-diameter CNT), there is no way to sustain this positive effect.

\section{$3.3 n$-Decane viscosity}

Whether from a theoretical [39], [56], [57] or experimental [58] perspective, calculating the viscosity of the liquid in such small-sized CNTs is full of challenges. The sliding of the layers required for shear flow is severely negatively affected by the CNT confinement in the radial direction [56], and the thermal fluctuations of the shear stress in the equilibrium state is therefore significantly different from that in the bulk system [57].

For a typical water/CNT system, when the diameter of the CNT is less than $3 \mathrm{~nm}$, the elongated tubular structure seriously affects the arrangement and movement of water molecules, resulting in the radial viscosity [56] of water being small enough to be ignored.

It is clear that the orientational ordering of $n$-decane molecules is more likely to be parallel to the axis of CNTs. Especially when the curvature is very high, the molecules desperately 
hope to avoid bending caused by the curvature of the wall in the radial direction. So, we use the Stokes-Einstein relationship to predict the $n$-decane viscosity, which is an attempt from the perspective of the axial self-diffusion coefficient.

The relationship between $n$-decane viscosity and curvature of CNTs at temperatures between $300 \mathrm{~K}$ and $360 \mathrm{~K}$ is presented in Fig. 6, which is diametrically opposite to the change of the axial self-diffusion coefficient of $n$-decane. The viscosity of $n$-decane does not decrease monotonically with the decrease of pore size. Comparing this trend with several MD results for water viscosity, some of which show similar changes [39], [57], while others are different [28], [56].

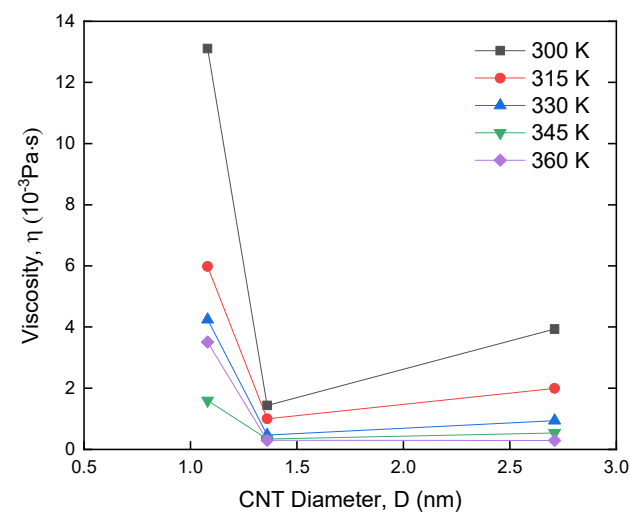

(a)

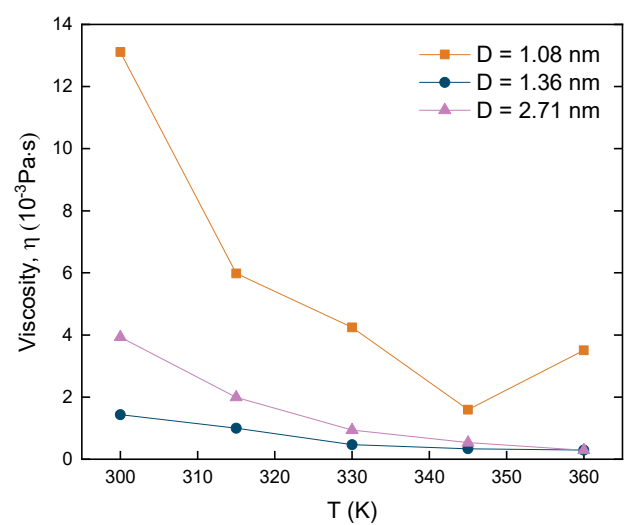

(b)

Figure 6: Curvature and temperature effect on $n$-decane viscosity, as predicted from equilibrium MD simulation using eqn (7). (a) Viscosity versus CNT diameter and (b) Viscosity versus temperature.

The raised temperature cannot change the non-monotonic variation between the curvature and the viscosity (Fig. 6(a)). And for individual CNTs, the relationship between temperature and $n$-decane viscosity is relatively simple (Fig. 6(b)), i.e. in general, increasing the temperature will cause a decrease in $n$-decane viscosity. It is important to note that for the $1.08 \mathrm{~nm}$-diameter CNT, this trend will change when the effect of temperature dominates.

\section{CONCLUSIONS}

More significant curvature and temperature changes can seriously affect the diffusion coefficient and viscosity of $n$-decane, as well as the friction coefficient between it and the wall of CNT. The OPLS model and Lennard-Jones potential is used to describe the intermolecular/intramolecular interactions in a typical $n$-decane/CNT system. All MD simulations are conducted in the NVT ensemble to show the dynamic of $n$-decane molecules in $1.08,1.36$, and $2.71 \mathrm{~nm}$-diameter single-walled armchair CNTs.

Increased curvature causes the $n$-decane/CNT friction coefficient to decline rapidly; however, the changes in the axial self-diffusion coefficient and viscosity are non-monotonic. On the contrary, the effect of increasing temperature is just the opposite, that is, for individual CNTs, the axial self-diffusion coefficient generally increases, and the viscosity decreases, but the friction coefficient fluctuates. 
The change in temperature makes the tripartite game among the curvature, the depletion area at the $n$-decane/CNT interface and the central area a little more complicated, especially for the $1.08 \mathrm{~nm}$-diameter CNT. Therefore, it is worth emphasizing that even with high temperatures, a CNT with a more significant curvature does not mean that $n$-decane is more difficult to transport through.

\section{ACKNOWLEDGEMENTS}

This work was financially supported by the Beijing Natural Science Foundation (Grant No. 3212020) and the National Natural Science Foundation of China (Grant No. 52004303).

\section{REFERENCES}

[1] Schoch, R.B., Han, J. \& Renaud, P., Transport phenomena in nanofluidics. Rev. Mod. Phys, 80, p. 839, 2008.

[2] Bocquet, L. \& Charlaix, E., Nanofluidics from bulk to interfaces. Chem. Soc. Rev, 39, p. 1073, 2010.

[3] Dong, X., Liu, H., Guo, W., Hou, J., Chen, Z. \& Wu, K., Study of the confined behavior of hydrocarbons in organic nanopores by the potential theory. Fluid Phase Equilib., 429, p. 214, 2016.

[4] Bocquet, L. \& Tabeling, P., Physics and technological aspects of nanofluidics. Lab Chip, 14, p. 3143, 2014.

[5] Hirai, Y. et al., Molecular dynamics studies on mechanical properties of carbon nano tubes with pinhole defects. Jpn. J. Appl. Phys., 42, p. 4120, 2003.

[6] Dong, X., Liu, H., Hou, J., Wu, K. \& Chen, Z., Phase equilibria of confined fluids in nanopores of tight and shale rocks considering the effect of capillary pressure and adsorption film. Ind. Eng. Chem. Res., 55, p. 798, 2016.

[7] Allen, M.P. et al., Introduction to molecular dynamics simulation. Comput. Soft Matter from Synth. Polym. to Proteins, 23, p. 1, 2004.

[8] Ma, M.D., Shen, L., Sheridan, J., Liu, J.Z., Chen, C. \& Zheng, Q., Friction of water slipping in carbon nanotubes. Phys. Rev. E, 83, p. 36316, 2011.

[9] Wang, L., Dumont, R.S. \& Dickson, J.M., Nonequilibrium molecular dynamics simulation of pressure-driven water transport through modified CNT membranes. $J$. Chem. Phys., 138, p. 124701, 2013.

[10] Babu, J.S. \& Sathian, S.P., Combining molecular dynamics simulation and transition state theory to evaluate solid-liquid interfacial friction in carbon nanotube membranes. Phys. Rev. E, 85, p. 51205, 2012.

[11] Falk, K., Sedlmeier, F., Joly, L., Netz, R.R. \& Bocquet, L., Ultralow liquid/solid friction in carbon nanotubes: Comprehensive theory for alcohols, alkanes, OMCTS, and water. Langmuir, 28, p. 14261, 2012.

[12] Whitby, M., Cagnon, L., Thanou, M. \& Quirke, N., Enhanced fluid flow through nanoscale carbon pipes. Nano Lett., 8, p. 2632, 2008.

[13] Majumder, M., Chopra, N., Andrews, R. \& Hinds, B.J., Enhanced flow in carbon nanotubes. Nature, 438, p. 44, 2005.

[14] Tsimpanogiannis, I.N., Moultos, O.A., Franco, L.F.M., de M, M.B. Spera, M. Erdős, \& Economou I.G., Self-diffusion coefficient of bulk and confined water: A critical review of classical molecular simulation studies. Mol. Simul., 45, p. 425, 2019.

[15] Nie, G.X., Wang, Y. \& Huang, J.P., Shape effect of nanochannels on water mobility. Front. Phys., 11, p. 114702, 2016.

[16] Zheng, Y., Ye, H., Zhang, Z. \& Zhang, H., Water diffusion inside carbon nanotubes: Mutual effects of surface and confinement. Phys. Chem. Chem. Phys., 14, p. 964, 2012. 
[17] Striolo, A., The mechanism of water diffusion in narrow carbon nanotubes. Nano Lett., 6, p. 633, 2006.

[18] Zhang, F., Molecular dynamics studies of chainlike molecules confined in a carbon nanotube. J. Chem. Phys., 111, p. 9082, 1999.

[19] Jabbari, F., Rajabpour, A. \& Saedodin, S., Thermal conductivity and viscosity of nanofluids: A review of recent molecular dynamics studies. Chem. Eng. Sci., 174, p. 67, 2017.

[20] Ye, H., Zhang, H., Zhang, Z. \& Zheng, Y., Size and temperature effects on the viscosity of water inside carbon nanotubes. Nanoscale Res. Lett., 6, p. 87, 2011.

[21] Babu, J.S. \& Sathian, S.P., The role of activation energy and reduced viscosity on the enhancement of water flow through carbon nanotubes. J. Chem. Phys., 134, p. 194509, 2011.

[22] Vakili-Nezhaad, G., Al-Wadhahi, M., Gujrathi, A.M., Al-Maamari, R. \& Mohammadi, M., Effect of temperature and diameter of narrow single-walled carbon nanotubes on the viscosity of nanofluid: A molecular dynamics study. Fluid Phase Equilib., 434, p. 193, 2017.

[23] Einstein, A., Eine Neue Bestimmung Der Moleküldimensionen, ETH Zurich, 1905.

[24] Kumaresan, V. \& Velraj, R., Experimental investigation of the thermo-physical properties of water-ethylene glycol mixture based CNT nanofluids. Thermochim. Acta, 545, p. 180, 2012.

[25] Sahu, P.\& Ali, S.M., Curious characteristics of polar and nonpolar molecules confined within carbon nanotubes (CNT) of varied diameter: Insights from molecular dynamics simulation. J. Chem. Eng. Data, 62, p. 2307, 2017.

[26] Jakobtorweihen, S., Lowe, C.P., Keil, F.J. \& Smit, B., A novel algorithm to model the influence of host lattice flexibility in molecular dynamics simulations: Loading dependence of self-diffusion in carbon nanotubes. J. Chem. Phys., 124, p. 154706, 2006.

[27] Bocquet, L. \& Barrat, J.L., On the green-Kubo relationship for the liquid-solid friction coefficient. J. Chem. Phys., 139, p. 44704, 2013.

[28] Thomas, J.A., McGaughey, A.J.H. \& Kuter-Arnebeck, O., Pressure-driven water flow through carbon nanotubes: Insights from molecular dynamics simulation. Int. J. Therm. Sci., 49, p. 281, 2010.

[29] Mashl, R.J., Joseph, S., Aluru, N.R. \& Jakobsson, E., Anomalously immobilized water: A new water phase induced by confinement in nanotubes. Nano Lett., 3, p. 589, 2003.

[30] Allen, M.P. \& Tildesley, D.J., Computer Simulation of Liquids, Oxford University Press, 2017.

[31] Zwanzig, R.W., Nonequilibrium Statistical Mechanics, Oxford University Press, 2001.

[32] Helfand, E., Transport coefficients from dissipation in a canonical ensemble. Phys. Rev., 119, p. 1, 1960.

[33] Cheng, A. \& Merz, K.M., The pressure and pressure tensor for macromolecular systems. J. Phys. Chem., 100, p. 905, 1996.

[34] Falk, K., Coasne, B., Pellenq, R., Ulm, F.J. \& Bocquet, L., Subcontinuum mass transport of condensed hydrocarbons in nanoporous media. Nat. Commun., 6, p. 6949, 2015.

[35] Li, J.C.M. \& Chang, P., Self-diffusion coefficient and viscosity in liquids. J. Chem. Phys., 23, p. 518, 1955.

[36] Heyes, D.M., The Liquid State: Applications of Molecular Simulations, Chichester, 1998. 
[37] Jorgensen, W.L., Madura, J.D. \& Swenson, C.J., Optimized intermolecular potential functions for liquid hydrocarbons. J. Am. Chem. Soc., 106, p. 6638, 1984.

[38] Jorgensen, W.L. \& Tirado-Rives, J., The OPLS [Optimized potentials for liquid simulations] potential functions for proteins, energy minimizations for crystals of cyclic peptides and crambin. J. Am. Chem. Soc., 110, p. 1657, 1988.

[39] Falk, K., The Molecular Origin of Fast Fluid Transport in Carbon Nanotubes: Theoretical and Molecular Dynamics Study of Liqui/Solid Friction in Graphitic Nanopores, Université Claude Bernard - Lyon I, 2011.

[40] Nicolas, J.P. \& Smit, B., Molecular dynamics simulations of the surface tension of NHexane, n-Decane and n-Hexadecane. Mol. Phys., 100, p. 2471, 2002.

[41] Mundy, C.J., Siepmann, J.I. \& Klein, M.L., Calculation of the shear viscosity of decane using a reversible multiple time-step algorithm. J. Chem. Phys., 102, p. 3376, 1995.

[42] Smit, B., Karaborni, S. \& Siepmann, J.I., Computer simulations of vapor-liquid phase equilibria of N-alkanes. J. Chem. Phys., 102, p. 2126, 1995.

[43] Cui, S.T., Cummings, P.T. \& Cochran, H.D., Multiple time step nonequilibrium molecular dynamics simulation of the rheological properties of liquid N-decane. $J$. Chem. Phys., 104, p. 255, 1996.

[44] Supple, S. \& Quirke, N., Rapid imbibition of fluids in carbon nanotubes. Phys. Rev. Lett., 90, p. 214501, 2003.

[45] Humphrey, W., Dalke, A. \& Schulten, K., VMD: Visual molecular dynamics. J. Mol. Graph., 14, p. 33, 1996.

[46] Martyna, G.J., Klein, M.L. \& Tuckerman, M., Nosé-Hoover chains: The canonical ensemble via continuous dynamics. J. Chem. Phys., 97, p. 2635, 1992.

[47] Guillaud, E., Merabia, S., de Ligny, D. \& Joly, L., Decoupling of viscosity and relaxation processes in supercooled water: A molecular dynamics study with the TIP4P/2005f model. Phys. Chem. Chem. Phys., 19, p. 2124, 2017.

[48] Chen, T., Smit, B. \& Bell, A.T., Are pressure fluctuation-based equilibrium methods really worse than nonequilibrium methods for calculating viscosities?, J. Chem. Phys., 131, p. 246101, 2009.

[49] Nevins, D. \& Spera, F.J., Accurate computation of shear viscosity from equilibrium molecular dynamics simulations. Mol. Simul., 33, p. 1261, 2007.

[50] Han, Y., Alsayed, A.M., Nobili, M., Zhang, J., Lubensky, T.C. \& Yodh, A.G., Brownian motion of an ellipsoid. Science, 314, p. 626, 2006.

[51] Happel, J. \& Brenner, H., Low Reynolds Number Hydrodynamics: With Special Applications to Particulate Media, Kluwer Academic, 1983.

[52] Ferguson, A.L., Panagiotopoulos, A.Z., Debenedetti, P.G. \& Kevrekidis, I.G., Systematic determination of order parameters for chain dynamics using diffusion maps. Proc. Natl. Acad. Sci., 107, p. 13597, 2010.

[53] Liu, Y., Wang, Q., Wu, T. \& Zhang, L., Fluid structure and transport properties of water inside carbon nanotubes. J. Chem. Phys., 123, p. 234701, 2005.

[54] Joseph, S. \& Aluru, N.R., Why are carbon nanotubes fast transporters of water?, Nano Lett., 8, p. 452, 2008.

[55] Barati Farimani, A. \& Aluru, N.R., Spatial diffusion of water in carbon nanotubes: From fickian to ballistic motion. J. Phys. Chem. B, 115, p. 12145, 2011.

[56] Köhler, M.H. \& da Silva, L.B., Size effects and the role of density on the viscosity of water confined in carbon nanotubes. Chem. Phys. Lett., 645, p. 38, 2016. 
[57] Zaragoza, A., Gonzalez, M.A., Joly, L., López-Montero, I., Canales, M.A., Benavides, A.L. \& Valeriani, C., Molecular dynamics study of nanoconfined TIP4P/2005 water: How confinement and temperature affect diffusion and viscosity. Phys. Chem. Chem. Phys., 21, p. 13653, 2019.

[58] Mattia, D. \& Gogotsi, Y., Review: Static and dynamic behavior of liquids inside carbon nanotubes. Microfluid. Nanofluidics, 5, p. 289, 2008. 